\title{
НАРУШЕНИЯ СНА
}

КАК МЕЖДИСЦИПЛИНАРНАЯ ПРОБЛЕМА

\begin{abstract}
Инсомния представляет собой расстройство, которое значительно снижает качество жизни, влияет на работоспособность, обучаемость, может приводить к социальной дезадаптации, личностным изменениям, а также связано с рядом метаболических нарушений. Подчеркивается важность междисциплинарного подхода к лечению инсомнических расстройств, обсуждаются принципы использования снотворных препаратов для коррекции нарушений сна. Приводятся результаты применения мелатонина у 2062 пациентов в возрасте 45-70 лет.
\end{abstract}

Ключевые слова: нарушения сна, инсомния, лечение, мелатонин.

E.Y. YAKUPOV, MD, Prof., Kazan State Medical University, MoH RF

SLEEP DISORDERS: AN INTERDISCIPLINARY PROBLEM

Insomnia is a sleep disorder that significantly reduces the quality of life, affects performance, learning ability, may lead to social maladjustment, personality changes, and is associated with a number of metabolic disorders. The article emphasizes the importance of an interdisciplinary approach to the treatment of sleep disorders, discusses administration of sleeping pills to manage sleep disorders.

Keywords: sleep disorders, insomnia, treatment, melatonin.

(3) начимость нарушений сна для регуляции жизнедеятельности человека общеизвестна. Пожалуй, нет специалиста в любой медицинской сфере, который бы не слышал от своих пациентов жалоб на бессонницу или плохой сон. В то же время и среди лиц, относящих себя к здоровым людям, зачастую можно услышать жалобы на нарушения сна. В общей популяции от 20 до 25\% взрослых страдают различными проявлениями инсомнии. Причем, по данным ряда авторов, эти нарушения носят выраженный и хронический характер: так, у более половины людей пожилого возраста выявляется хроническая инсомния. Наконец, только 30\% лиц, страдающих бессонницей, обращаются к врачу! Безусловно, проблема нарушений сна стара как мир - можно привести немало примеров от Античности до наших дней, когда и сон и его нарушения рассматривались как нечто загадочное, трудно поддающееся коррекции и лечению. Вот лишь одна выдержка из статьи в British Medical Jornal от 1896 г.: «...Бессонница снова является объектом пристального внимания общества. В большинстве случаев причиной бессонницы является постоянное напряжение свойственное современной жизни. Статьи и письма полны хороших советов, как можно жить более спокойно, и общих фраз о том, как вредно волноваться. Но, к сожалению, очень многие люди не могут последовать этим советам и ведут жизнь полную тревоги и беспокойства...».

По данным ряда современных исследований, в целом отмечено уменьшение длительности ночного сна. Например, в американской популяции в 2005 г. продолжительность ночного сна у $40 \%$ взрослых была менее 7 ч, в то время как в 1960 г. этот показатель не превышал 15\% [7, 10]. Причем недостаточный сон сопровождается пониженной работоспособностью, ухудшением познавательной функции, здоровья и качества жизни. До 75\% взрослых жалуется на беспокойный сон, пробуждение ночью и храп, хотя большинство игнорирует эту проблему. Только 25\% американцев отметили, что проблемы со сном отрицательно влияют на их дневную жизнь $[7,10]$. Причем 65\% заявили, что их лечащий врач никогда не спрашивал их о том, как они спят! Таким образом, мы можем видеть, что, несмотря на широкую представленность нарушений сна в общей популяции, до сих пор не уделяется должного внимания этой проблеме.

\section{Инсомния обусловливает значительное снижение качества жизни, социальную дезадаптацию, недостаточную работоспособность, обучаемость и даже личностные изменения}

Инсомния представляет собой расстройство, которое проявляется нарушениями качества, количества или времени сна, необходимых для нормальной активной деятельности. Эта патология обусловливает значительное снижение качества жизни, социальную дезадаптацию, недостаточную работоспособность, обучаемость и даже личностные изменения. Кроме того, изменения биохимических параметров организма при недосыпании непосредственно связаны с рядом метаболических нарушений.

Пациенты при обращении к врачу ограничиваются зачастую скудными формулировками, говоря лишь о пло- 
хом сне или бессоннице. Между тем насчитывают около 100 симптомов нарушений сна, которые группируются в гиперсомнии, инсомнии, парасомнии и расстройства суточного ритма «сон - бодрствование». Для получения наиболее полного представления о нарушениях сна необходимо выяснить целый ряд характеристик: общая продолжительность сна, период засыпания, глубина сна. Очень важны собственные представления пациента о его сне, сопутствующих ощущениях, самочувствии в ночные часы и после утреннего подъема. Все это помогает в определенной мере определить и форму нарушений сна и их этиологию.

Этиология инсомний может быть самой разнообразной. Согласно МКБ-10, расстройства сна в основном первично психогенные, эмоционально обусловленные состояния [1].

Причины инсомнии, как правило, определяют ее длительность. Эпизодическая инсомния длительностью до 1 недели чаще всего возникает вследствие эмоциональных стрессов, десинхроноза, неправильного режима дня, возникновения соматической патологии. Она может быть обусловлена внешними, нарушающими сон факторами (шум, свет, колебания комнатной температуры и др.), к которым некоторые люди имеют повышенную чувствительность. В большей степени астенизирует пациента кратковременная инсомния, которая длится 2-3 недели. Ее причинами являются расстройства адаптации, тяжелые и продолжительные стрессовые ситуации, обострения хронических соматических заболеваний, хронический болевой синдром, различные кожные заболевания с зудом, ночной миотонус и др. Причинами хронической инсомнии (более 3 недель) чаще всего являются психические расстройства, злоупотребление психоактивными веществами, прием стимулирующих психотропных средств и других лекарственных препаратов, среди побочных действий которых нередко отмечаются и инсомнии.

По времени появления нарушения сна могут быть пре-, интра- и постсомническими. У 80\% пациентов отмечаются пресомнические расстройства, т. е. нарушения засыпания с запаздыванием наступления сна до 2 ч и более, общей продолжительностью сна около 4 ч, хотя в постели человек проводит 7-8 ч. Когда пациент не может уснуть, его, как правило, беспокоят какиелибо навязчивые воспоминания, тревожные опасения, он мысленно «проигрывает» какие-то ситуации. Утром пациент чувствует сонливость и слабость. Примерно у 30\% пациентов встречаются интрасомнические нарушения в виде внезапных пробуждений (в страхе, с вегетативными нарушениями) или поверхностного сна, наполненного грезами. Общая продолжительность сна может составлять всего 2-3 ч, после которых пациент также не ощущает утренней бодрости и свежести. К постсомническим нарушениям относится каталепсия генетически обусловленное заболевание, проявляющееся дневной сонливостью.

Крайне важным аспектом является междисциплинарный характер инсомнии. По нашему глубокому убеждению, ее надо рассматривать не только как неврологическую проблему, но и в целом, в рамках клинической медицины, актуализируя ее и для врачей терапевтического профиля. Как эпидемиологические, так и физиологические исследования обнаружили связь между сокращением времени сна и отдельными составляющими метаболического синдрома X, причем эта связь двунаправлена $[6,9,14]$. Плохой сон увеличивает риск возникновения или обострения метаболических или сердечно-сосудистых нарушений, а эта патология, в свою очередь, увеличивает риск инсомнии. Так, известна прямая коррелятивная связь между сном и ожирением, хотя ее механизмы не до конца изучены. По данным краткосрочных физиологических исследований, лишение сна на 4 ч за ночь в течение 1 недели ведет к значительному уменьшению лептина и повышению грелина, к стимуляции чувства голода и аппетита [13]. Возможно, повышение аппетита в условиях недостаточного сна может частично стимулировать развитие ожирения.

\section{Эпидемиологические и физиологические исследования установили связь между сокращением времени сна и отдельными составляющими метаболического синдрома $X$}

Кардиоваскулярные факторы риска, например артериальная гипертония и дислипидемия, связаны с высокими уровнями нетрудоспособности и смертности. Суточный мониторинг артериального давления (АД) у мужчин показал значимое повышение АД после короткого ночного сна (3-6 ч) по сравнению с длинным (8 4) [15]. Это повышение АД обусловлено активизацией симпатоадреналовой системы [12]. Данные другого эпидемиологического анализа свидетельствуют о возрастании гиперхолестеринемии на фоне недостаточного сна (< 5 ч по сравнению с 7-часовым ночным сном) [6]. Кроме того, по данным 10-летнего когортного исследования, короткий сон (<5 ч по сравнению с 8-часовым ночным сном) в 1,45 раза увеличивает риск возникновения ишемии миокарда [6] Японские исследователи отметили, что ночной сон менее 5 ч в 2-3 раза увеличивает риск инфаркта миокарда [8].

Все вышесказанное диктует необходимость изучения проблемы нарушений сна с точки зрения выявления причинно-следственных связей. Конечно, существуют специальные методы диагностики-полисомнография, актиграфия, использование специфических опросников. Но даже внимательный сбор анамнеза, анализ жалоб пациента страдающего от инсомнии позволит не только правильно «подойти» к диагнозу, но и подобрать адекватную лечебную стратегию.

Традиционно для лечения инсомнических расстройств применяются снотворные препараты [1, 3, 5, 19]. Определен ряд принципов назначения снотворных препаратов. Так, начинать лечение инсомний следует с 
растительных снотворных средств - наиболее естественных препаратов, не нарушающих химизм клеток. Предпочтительными являются короткоживущие препараты, позволяющие избежать появления утренней вялости, слабости. Длительность терапии снотворными препаратами должна составлять не более 3 недель, оптимально - 10-14 дней (в связи с привыканием человека к медикаментозной поддержке сна). Пожилым людям назначается половинная суточная доза снотворных средств, к тому же учитывается возможность взаимодействия с другими препаратами. При необходимости длительного назначения снотворных необходимо помнить о риске возникновения синдрома апноэ во сне и проводить так называемые «лекарственные каникулы», когда организм отдыхает от снотворных средств и пытается самостоятельно справиться с нарушениями сна.

Практический опыт лечения инсомний позволил сформулировать свойства эффективного гипнотика, который должен способствовать быстрому наступлению сна, его поддержанию, препятствовать частым пробуждениям, а при их возникновении - позволять снова быстро уснуть. Эффективный гипнотик после утреннего пробуждения не оставляет ощущения вялости, разбитости, усталости; не оказывает побочного действия на пациента и, что самое важное, не ухудшает социальной адаптации больного.

Долгое время препаратами первой линии при нарушениях сна были барбитураты. Сейчас ВО3 не рекомендует применение этих препаратов в качестве снотворных средств, учитывая их побочные действия, формирование зависимости, существенное нарушение структуры сна и другие факторы. Однако пациенты достаточно часто принимают барбитураты, причем нередко в высоких дозах и на протяжении длительного времени.

\section{Длительность терапии снотворными препаратами не должна превышать 3 недель, оптимально - 10-14 дней (в связи с привыканием человека к медикаментозной поддержке сна)}

В качестве снотворных средств ранее достаточно широко использовались бензодиазепины. Однако среди производных бензодиазепина для терапии нарушений сна могут применяться только препараты короткого действия. Бензодиазепины увеличивают общее время сна и длительность второй стадии фазы медленного сна, уменьшают двигательную активность во сне, снижают время засыпания. Значительным недостатком бензодиазепинов является развитие привыкания и зависимости, что обусловливает необходимость увеличения дозы; отмечается синдром отмены. При употреблении препаратов этой группы возрастает риск возникновения апноэ вследствие миорелаксирующего эффекта. Бензодиазепины вызывают интенсификацию сна, поэтому пациент утром чувствует себя «разбитым».
При инсомниях также используют моноамины, которые не менее эффективны, чем бензодиазепины, но имеют некоторое дневное последействие и ряд противопоказаний (возраст до 16 лет, глаукома, аденома простаты), поэтому применение этих препаратов несколько ограничено.

\section{Эффективный гипнотик не вызывает ощущения вялости, разбитости, усталости после утреннего пробуждения; не оказывает побочного действия, не ухудшает сочиальной адаптации больного}

Сейчас весьма популярны гипнотики III поколения зопиклон и золпидем. Они достаточно эффективны и безопасны, поэтому широко применяются в клинической практике. Однако врачи и ученые не теряют надежды найти еще более безопасные, физиологические способы коррекции нарушений сна, на это же ориентированы и наши пациенты (всем хорошо известны не всегда безосновательные опасения многих больных по поводу употребления «химии»). В связи с этим особого внимания заслуживают препараты мелатонина, действие которого определяется его способностью регулировать циркадные ритмы, биологическую смену сна и бодрствования посредством сложных нейрогуморальных механизмов центральной нервной системы.

Мелатонин - нейрогормон, продуцируемый в темное время суток эпифизом (до 80\%), сетчаткой и цилиарным телом глаза, энтерохромаффинными клетками желудочно-кишечного тракта. Мелатонин регулирует суточные и сезонные ритмы организма, основным из которых является цикл «бодрствование - сон», модулирует активность эндокринных желез (гипофиза, надпочечников, половых, щитовидной и вилочковой желез), обеспечивает устойчивость организма к стрессовым воздействиям, связывает свободные радикалы и повышает активность антиоксидантных ферментов [2, 9, 11, 14, 16-18]. Интересно, что у больных с психическими нарушениями есть свои особенности в синтезе мелатонина. Так, при депрессии синтез мелатонина может продолжаться до полудня, при астении он хаотичен в течение суток, при маниях и других видах гиперактивности пациента выработка мелатонина снижена, при шизофрении - повышена. Нарушение суточного ритма синтеза мелатонина, которое наблюдается при нарушениях сна, приводит к развитию истерических состояний, раздражительности, снижению памяти, концентрации внимания, ухудшению настроения, вегетативным дисфункциям.

Имеются данные о том, что нарушение синтеза мелатонина ассоциируется с увеличением веса и склонностью к ожирению (поскольку существует взаимосвязь синтеза мелатонина с обменом кортизола и инсулина). Кроме того, при снижении синтеза мелатонина увеличивается содержание холестерина и триглицеридов в крови. Таким образом, можно полагать, что нарушения синтеза мелатонина непосредственно связаны с развитием метаболического синдрома $[6,9,18]$. 


\section{КОРРЕКЦИЯ ЦИКЛА СОН-БОДРСТВОВАНИЕ ПРИ НЕВРОЛОГИЧЕСКИХ РАССТРОЙСТВАХ}

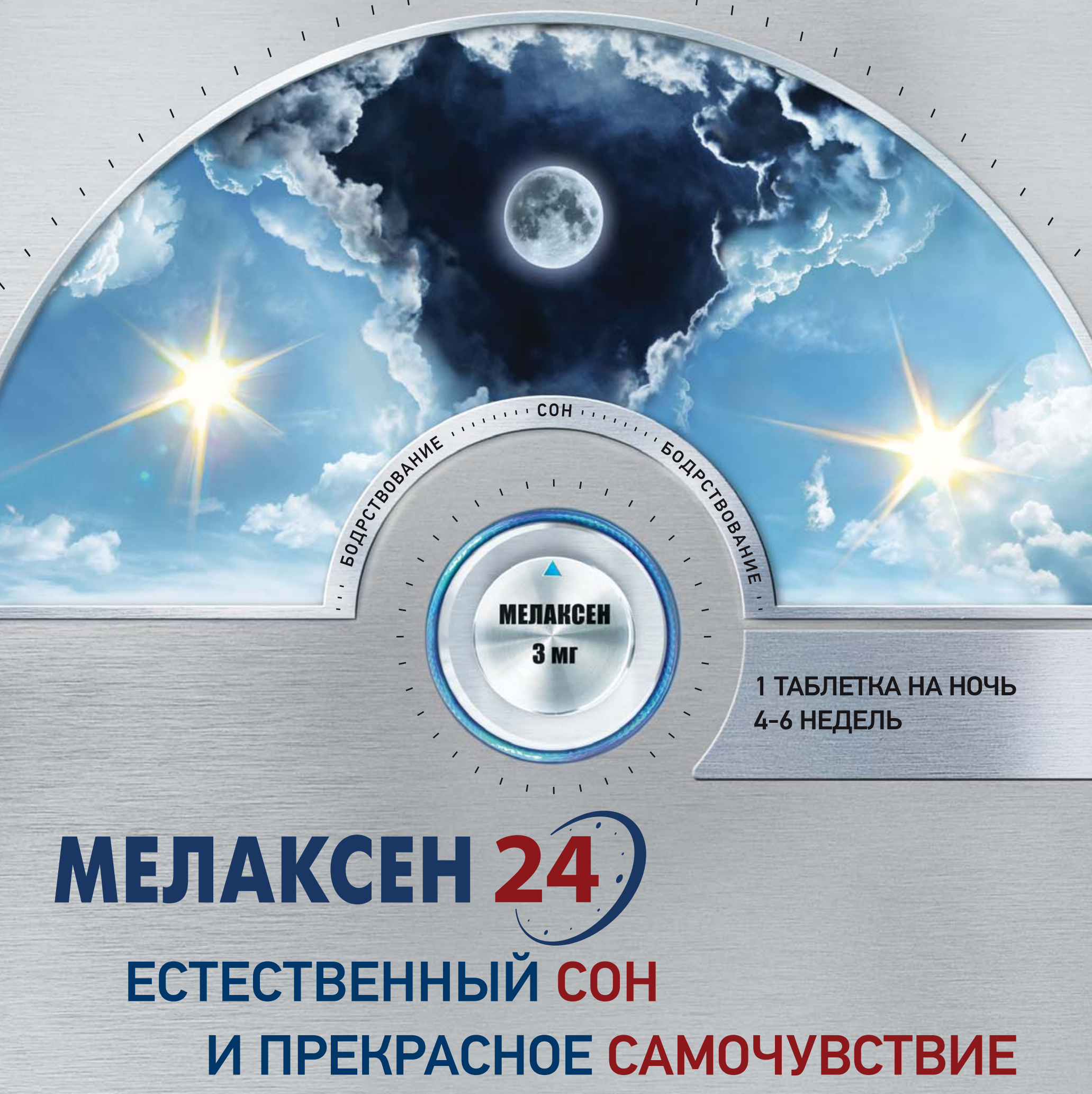

Рег. Номер - П N015325/01 290808 Представительство компании «Юнифарм, Инк.» (США): 115162, Москва, ул. Шаболовка, д. 31, стр. Б, Т./факс: +7 (495) 995 7767. www.unipharm.ru 
Мелатонин снижает активность гипофизарно-симпатоадреналовой системы, тем самым уменьшая выраженность начальной фазы стрессовой реакции, т. е. повышает устойчивость организма к стрессовым влияниям. Таким образом, препараты мелатонина применяются при нарушениях адаптации, проявляющихся ухудшением сна.

\section{Мелатонин регулирует суточные и сезонные ритмы организма, основным среди которых является цикл «бодрствование - сон», модулирует активность эндокринных желез (гипофиза, надпочечников, половых, щитовидной и вилочковой желез), обеспечивает устойчивость организма к стрессовым воздействиям, связывает свободные радикалы и повышает активность антиоксидантных ферментов}

В 2011 г. в 22 медицинских центрах РФ было проведено обсервационное исследование по оценке терапевтической эффективности и переносимости препарата Мелаксен (мелатонин), назначаемого в стандартных дозах в рутинной клинической практике по поводу нарушений сна по типу инсомнии пациентам с хронической церебральной сосудистой недостаточностью. Использовался препарат Мелаксен в таблетках, содержащих 3 мг мелатонина. Длительность непрерывной терапии Мелаксеном составила 24 дня. В исследование были включены 2062 пациента в возрасте 45-70 лет, страдающие различными формами хронической церебральной сосудистой недостаточности по критериям МКБ-10 или дисциркуляторной энцефалопатии, оформившие письменное информированное согласие пациента на участие в обсервационном исследовании.

Мелаксен назначался в соответствии с Инструкцией по медицинскому применению препарата. Всем пациентам Мелаксен назначается в терапевтической дозе 3 мг за 40 мин до укладывания в постель.

При включении в исследование все пациенты заполняли протокол исследования больных с нарушениями сна для уточнения антропометрических показателей, анамнеза, характера и длительности нарушений сна, сведений об особенностях цикла сон-бодрствование, принимаемых препаратах; проводилась клиническая оценка соматического и неврологического статуса.

Эффективность терапии Мелаксеном оценивалась с помощью анкеты балльной оценки субъективных характеристик сна, госпитальной шкалы тревоги и депрессии (HADS), анкеты скрининга синдрома апноэ во сне, шкалы сонливости (Epworth). Безопасность/ переносимость терапии Мелаксена оценивались на основании зарегистрированных в ходе исследования нежелательных явлений (НЯ)/нежелательных реакций (HР). Предполагалось, что все НЯ, возникающие в про- цессе исследования, будут анализироваться с указанием их частоты, вида, серьезности и причинной связи с исследуемым препаратом.

Основными выводами по столь масштабному исследованию, возможно, впервые проводившемуся в нашей стране по проблеме нарушений сна были следующие:

1. Прием Мелаксена в дозе 3 мг/сут у больных хронической церебральной сосудистой недостаточностью с инсомнией приводил к улучшению субъективно оцениваемого качества ночного сна.

2. Улучшение сна отмечается уже через 2 недели приема препарата, в последующие 10 дней отмечается дальнейшее улучшение показателей сна.

3. В наибольшей степени на фоне приема Мелаксена уменьшается число ночных пробуждений, также наблюдается значительное укорочение времени засыпания, улучшение качества утреннего пробуждения и общего качества сна. В меньшей степени на фоне лечения уменьшается частота сновидений.

4. На фоне лечения Мелаксеном отмечается уменьшение выраженности проявлений апноэ во сне по данным соответствующей анкеты.

5. Улучшение сна на фоне приема препарата сопровождается уменьшением выраженности дневной сонливости, депрессии и тревоги.

6. Показатели сонливости, выраженности проявлений апноэ во сне, тревоги и депрессии улучшаются уже через 2 недели лечения и продолжают улучшаться в течение последующих 10 дней.

7. Положительный эффект Мелаксена на сон не зависит от пола, возраста, выраженности проявлений апноэ во сне, уровня сонливости, степени выраженности тревоги и депрессии, продолжительности нарушений сна, причины нарушения сна.

8. Применение Мелаксена в дозе 3 мг в течение 24 дней безопасно, по мнению пациентов, принимавших препарат, и по мнению наблюдавших их врачей.

\section{На фоне приема Мелаксена уменьшается число ночных пробуждений, наблюдается значительное укорочение времени засыпания, улучшение качества утреннего пробуждения и общего качества сна}

Таким образом, резюмируя представленные данные, следует рассматривать инсомнические расстройства через призму междисциплинарных подхода с актуальным использованием всего арсенала диагностических возможностей врача. Памятуя о широком диапазоне лечебных стратегий, в которых особое место занимают поведенческие методики и взвешенное отношение к фармакологическим препаратам, мы не должны забывать о «мягком» подходе в дебюте лечения пациентов с нарушениями сна, в связи с чем использование Мелаксена у этой группы больных занимает особое место. 


\section{ЛИТЕРАТУРА:}

1. Morin CM, Hauri PJ, Espie CA, Spielman AJ, Buysse DJ, Bootzin RR. Nonpharmacologic treatment of chronic insomnia: an American Academy of Sleep Medicine review. Sleep, 1999, 22: 1134-56.

2. Costa e Silva JA, Chase M, Sartorius M, Roth T. Special report from a symposium held by the World Health Organization and the World Federation of Sleep Research Societies: an overview of insomnias and related disorders recognition, epidemiology, and rational management. Sleep, 1996, 19: 412-6.

3. The international classification of sleep disorders, revised: diagnostic and coding manual. Rochester, Minn.: American Sleep Disorders Association, 1997.

4. Morin CM, Culbert JP, Schwartz SM. Nonpharmacological interventions for insomnia: a meta-analysis of treatment efficacy. Am J Psychiatry, 1994, 151: 1172-80.

5. Spielman AJ, Caruso LS, Glovinsky PB.A behavioral perspective on insomnia treatment. Psychiatr Clin North Am, 1987, 10: 541-53.

6. Littner M, Hirshkowitz M, Kramer M, et al. Practice parameters for using polysomnography to evaluate insomnia: an update. Sleep, 2003, 26: 754-60.

7. The international classification of sleep disorders: diagnostic \& coding manual, ICDS-2. $2^{\text {nd }}$ ed. Westchester, Ill.: American Academy of Sleep Medicine, 2005.

8. Perlis ML, Smith MT, Pigeon WR. Etiology and pathophysiology of insomnia. In: KrygerMH, RothT, Dement WC, eds. Principles and practice of sleep medicine. 4th ed. Philadelphia: Elsevier/Saunders, 2005: 714-25.

9. Bootzin RR, Epstein D, Wood JM. Stimulus control instructions. In: Hauri PJ, ed. Case studies in insomnia. New York: Plenum Medical Book, 1991: 19-28.

10. Spielman AJ, Saskin P, Thorpy MJ. Treatment of chronic insomnia by restriction of time in bed. Sleep, 1987, 10: 45-56.

11. Hauri PJ. Sleep hygiene, relaxation therapy, and cognitive interventions. In: Hauri PJ, ed. Case studies in insomnia. New York: Plenum Medical Book, 1991: 65-84.

12. Murtagh DRR, Greenwood KM. Identifying effective psychological treatments for insomnia: a meta-analysis. J Consult Clin Psychol, 1995, 63: 79-89.

13. Espie CA, Inglis SJ, Tessier S, Harvey L. The clinical effectiveness of cognitive behaviour therapy for chronic insomnia: implementation and evaluation of a sleep clinic in general medical practice. Behav Res Ther, 2001, 39: 4-60.

14. Edinger JD, Wohlgemuth WK, Radtke RA, Marsh GR, Quillian RE. Cognitive behavioral therapy for treatment of chronic primary insomnia: a randomized controlled trial. JAMA, 2001, 285: 1856-64.

15. Turner RM, Ascher LM. Therapist factor in the treatment of insomnia. Behav Res Ther, 1982 20: $33-40$

16. Morgan K, Dixon S, Mather N, Thompson J, Tomeny M. Psychological treatment for insomnia in the management of long-term hypnotic drug use: a pragmatic randomised controlled trial. Br J Gen Pract, 2003, 53: 923-8.
17. Baillargeon L, Demers L, Ladouceur R. Stimulus-control: nonpharmacologic treatment for insomnia. Can Fam Physician, 1998, 44: 73-9.

18. Edinger JD, Sampson WS. A primary care «friendly» cognitive behavioral insomnia therapy. Sleep, 2003, 26: 177-82.

19. Riedel BW, Lichstein KL, Dwyer WO. Sleep compression and sleep education for older insomniacs: self-help versus therapist guidance. Psychol Aging, 1995, 10: 54-63.

20. Mimeault V, Morin CM. Self-help treatment for insomnia: bibliotherapy with and without professional guidance. J Consult Clin Psychol, 1999 67: 511-9.

21. Meoli AL, Rosen CL, Kristo D, et al. Oral nonpre scription treatment for insomnia: an evaluation of products with limited evidence. J Clin Sleep Med, 2005, 1: 173-87.

22. Rogers NL, Dinges DF, Kennaway DJ, Dawson D. Potential action of melatonin in insomnia. Sleep, 2003, 26: 1058-9.

23. Nowell PD, Mazumdar S, Buysse DJ, Dew MA, Reynolds CF III, Kupfer DJ. Benzodiazepines and zolpidem for chronic insomnia: a meta-analysis of treatment efficacy. JAMA, 1997, 278: 2170-7.

24. Holbrook AM, Crowther R, Lotter A, Cheng C, King D. Meta-analysis of benzodiazepine use in the treatment of insomnia. CMAJ, 2000, 162: 225-33.

25. Elie R, Ruther E, Farr I, Elilien G, Salinas E. Sleep latency is shortened during 4 weeks of treatment with zaleplon, a novel nonbenzodiazepine hypnotic. J Clin Psychiatry, 1999, 60: 536-44.

26. Walsh JK, Vogel GW, Scharf M, et al. A five week, polysomnographic assessment of zaleplon 10 $\mathrm{mg}$ for the treatment of primary insomnia. Sleep Med, 2000, 1: 41-9.

27. Walsh JK, Pollak CP, Scharf MB, Schweitzer PK, Vogel GW. Lack of residual sedation following middle-of-the-night zaleplon administration in sleep maintenance insomnia. Clin Neuropharmacol, 2000, 23: 17-21.

28. Krystal AD, Walsh JK, Laska E, et al. Sustained efficacy of eszopiclone over 6 months of nightly treatment: results of a randomized, doubleblind, placebo-controlled study of adults with chronic insomnia. Sleep, 2003, 26: 793-9.

29. Walsh JK, Roth T, Randazzo A, et al. Eight weeks of non-nightly use of zolpidem for primary insomnia. Sleep, 2000, 23: 1087-96.

30. Perlis ML, McCall WV, Krystal AD, Walsh JK. Long-term, non-nightly administration of zolpidem in the treatment of patients with primary insomnia. J Clin Psychiatry, 2004, 65: 1128-37.

31. Voshaar RC, van Balkom AJ, Zitman FG Zolpidem is not superior to temazepam with respect to rebound insomnia: a controlled study. Eur Neuropsychopharmacol, 2004, 14 301-6.

32. Vogel GW, Morris D. The effects of estazolam on sleep, performance, and memory: a longterm sleep laboratory study of elderly insomniacs. J Clin Pharmacol, 1992, 32: 647-51.

33. Roth T, Roehrs TA. A review of the safety profiles of benzodiazepine hypnotics. J Clin Psychiatry, 1991, 52(Suppl): 38-41.

34. Monti JM, Attali P, Monti D, Zipfel A, de la Giclais B, Morselli PL. Zolpidem and rebound insomnia - a double-blind, controlled polysomnographic study in chronic insomniac patients. Pharmacopsychiatry, 1994, 27: 166-75.

35. Soldatos CR, Dikeos DG, Whitehead A. Tolerance and rebound insomnia with rapidly eliminated hypnotics: a meta-analysis of sleep laboratory studies. Int Clin Psychopharmacol, 1999, 14: 287-303.

36. Greenblatt DJ, Harmatz JS, Zinny MA, Shader RI. Effect of gradual withdrawal on the rebound sleep disorder after discontinuation of triazolam. N Engl J Med, 1987, 317: 722-8.

37. ScharfMB, Roth T, Vogel GW, Walsh JK. A multicenter, placebo-controlled study evaluating zolpidem in the treatment of chronic insomnia. J Clin Psychiatry, 1994, 55: 192-9.

38. Rothschild AJ. Disinhibition, amnestic reactions, and other adverse reactions secondary to triazolam: a review of the literature. J Clin Psychiatry, 1992, 53(Suppl): 69-79.

39. Roth T, Hartse KM, Saab PG, Piccione PM, Kramer $\mathrm{M}$. The effects of flurazepam, lorazepam, and triazolam on sleep and memory. Psychopharmacology (Berl), 1980, 70: 231-7.

40. Canaday BR. Amnesia possibly associated with zolpidem administration. Pharmacotherapy, 1996, 16: 687-9.

41. Morgenthaler TI, Silber MH. Amnestic sleeprelated eating disorder associated with zolpidem. Sleep Med, 2002, 3: 323-7.

42. Mendelson WB. Clinical distinctions between long-acting and short-acting benzodiazepines. J Clin Psychiatry, 1992, 53(Suppl): 4-7.

43. Morin CM, Colecchi C, Stone J, Sood R, Brink D. Behavioral and pharmacological therapies for late-life insomnia: a randomized controlled trial. JAMA, 1999, 281: 991-9.

44. Walsh JK, Schweitzer PK. Ten-year trends in the pharmacological treatment of insomnia. Sleep, 1999, 22: 371-5.

45. Kaynak H, Kaynak D, Gozukirmizi E, GuilleminaultC. The effects of trazodone on sleep in patients treated with stimulant antidepressants. Sleep Med, 2004, 5: 15-20.

46. Nierenberg AA, Adler LA, Peselow E, Zornberg G, Rosenthal M. Trazodone for antidepressantassociated insomnia. Am J Psychiatry, 1994, 151 : 1069-72.

47. Walsh JK, Erman M, Erwin CW, et al. Subjective hypnotic efficacy of trazodone in DSM3-R primary insomnia. Hum Psycho pharmacol, 1998, 13: 191-8.

48. Hajak G, Rodenbeck A, Voderholzer U, et al. Doxepin in the treatment of primary insomnia: a placebo-controlled, double-blind, polysomnographic study. J Clin Psychiatry, 2001, 62: 45363

49. Aslan S, Isik E, Cosar B. The effects of mirtazapine on sleep: a placebo controlled, doubleblind study in young healthy volunteers. Sleep, 2002, 25: 677-9.

50. Hausser-Hauw et al. Effect on sleep architecture and residual effect of a dose of $15 \mathrm{mg}$ of Doxylamine versus triazolam treatment in persistent sleep-onset insomnia. Sep Hop Paris, 1995, 71: no 23-24, 742-750.

51. Practice Parameters for the Psychological and Behavioral Treatment of Insomnia: An Update. An American Academy of Sleep Medicine Report. Sleep, 2006, 29: 650-9. 